\title{
Anisotropic Skyrmion Diffusion Controlled by Magnetic-Field-Induced Symmetry Breaking
}

\author{
Nico Kerber®, ${ }^{1,2}$ Markus Weißenhofer® ${ }^{3}$ Klaus Raab $\odot,{ }^{1}$ Kai Litzius $\odot,{ }^{1,2,4}$ Jakub Zázvorka $\odot,{ }^{1,5}$ \\ Ulrich Nowak $\odot{ }^{3}$ and Mathias Kläui® ${ }^{1,2, *}$ \\ ${ }^{1}$ Institut für Physik, Johannes Gutenberg-Universität Mainz, Mainz 55099, Germany \\ ${ }^{2}$ Graduate School of Excellence Materials Science in Mainz, Mainz 55128, Germany \\ ${ }^{3}$ Fachbereich Physik, Universität Konstanz, Universitätsstraße 10, Konstanz 78457, Germany \\ ${ }^{4}$ Department of Materials Science and Engineering, Massachusetts Institute of Technology, Cambridge, \\ Massachusetts 02139, USA \\ ${ }^{5}$ Institute of Physics, Faculty of Mathematics and Physics, Charles University, Ke Karlovu 5, Prague 121 16, \\ Czech Republic
}

(Received 22 February 2021; revised 8 March 2021; accepted 29 March 2021; published 20 April 2021)

The diffusion of particles has wide repercussions, ranging from particle-based soft-matter systems to solid-state systems with particular electronic properties. Recently, in the field of magnetism, the diffusion of magnetic skyrmions, topologically stabilized quasiparticles, has been demonstrated. Here, we show that, by applying a magnetic in-plane field, and therefore, breaking the symmetry of the system, skyrmion diffusion becomes anisotropic, with faster diffusion parallel to the field axis and slower diffusion perpendicular to it. We furthermore show that the absolute value of the applied magnetic in-plane field controls the absolute values of the diffusion coefficients, so that one can thereby tune both the orientation of the diffusion and its strength. Based on the stochastic Thiele equation, we can explain the observed anisotropic diffusion as a result of the elliptical deformation of the skyrmions by the application of the in-plane field.

DOI: 10.1103/PhysRevApplied.15.044029

\section{INTRODUCTION}

Magnetic skyrmions, topologically stabilized spin structures, allow for a wide range of promising roomtemperature applications in logic, data storage, probabilistic computing, and many other areas [1-6]. Skyrmions can be stabilized by the chiral Dzyaloshinskii-Moriya interaction $[7,8]$, arising in bulk systems with broken inversion symmetry, such as B20 compounds [9-11], as well as in thin multilayered films with structural inversion asymmetry [12-14]. It is demonstrated that it is possible to reproducibly write and delete single skyrmions [14,15]. Furthermore, reproducible skyrmion displacement in heavy metal-ferromagnet thin-film heterostructures by the highly efficient spin-orbit torques generated through the interface that also provide the homochiral character of the spin textures, has been thoroughly investigated [15-19]. While current-induced skyrmion dynamics is a major field of study in skyrmionics research, due to promising applications in storage and logic devices, studies investigating thermally induced skyrmion dynamics are elusive so far. This is frequently based on the common view that thermal

*klaeui@uni-mainz.de diffusion is strongly suppressed in magnetic systems in which damping is typically small [20]. It has been shown recently that, in specially tailored low-pinning multilayers, pure isotropic skyrmion diffusion at room temperature can be achieved [6,21-23]. By changing the sample temperature, one obtains control over the diffusion strength, since the diffusion coefficient depends exponentially on the temperature, in the case of a nonflat energy landscape, as present in the experimental samples [6]. However, to use skyrmion diffusion, one needs to control and, for instance, confine diffusion along a certain direction, which can then possibly enable the implementation of skyrmion-based Brownian circuits [24].

While diffusion of skyrmions is a recent finding, diffusion is well established in the field of soft matter, where the diffusive motion of particles [25] was observed in 1827 by Brown and was theoretically described by Einstein, who established a relation between the friction coefficient of a Brownian particle and its diffusion coefficient [26]. In extensive subsequent investigations, it was shown that uniaxially fabricated ellipsoid particles exhibited stronger diffusion parallel, rather than perpendicular, to their long axis, leading to anisotropic translational Brownian diffusion $[27,28]$. This suggests that one can control diffusion by changing the particle shape. Nevertheless, in soft-matter 
systems, one cannot change the particle shape after the experiment has started, so there is limited control during the experiment. However, in magnetic systems, a flexible dynamic change to the skyrmion shape can be envisaged as an approach that provides flexible dynamic control of skyrmion diffusion.

Thus, here, we explore the effects of applied magnetic fields as a means to control the diffusive motion of skyrmions in a $\mathrm{Co}_{20} \mathrm{Fe}_{60} \mathrm{~B}_{20}$-based thin-film metalmultilayer system with perpendicular magnetic anisotropy. By Kerr microscopy, we determine the diffusion properties under the application of a magnetic in-plane (IP) field oriented along a selected direction. We find that application of magnetic in-plane fields leads to anisotropic diffusion, allowing one to control the diffusion direction and amplitude over a wide range, which can be explained theoretically based on an extended Thiele equation by a combination of analytical and numerical calculations.

\section{SAMPLE AND SETUP}

The investigated $\mathrm{Ta}(5 \mathrm{~nm}) / \mathrm{Co}_{20} \mathrm{Fe}_{60} \mathrm{~B}_{20}(1 \mathrm{~nm}) / \mathrm{Ta}$ $(0.08 \mathrm{~nm}) / \mathrm{MgO}(2 \mathrm{~nm}) / \mathrm{Ta}(5 \mathrm{~nm})$ material stack is deposited on a $\mathrm{Si} / \mathrm{SiO}_{2}$ substrate via dc-magnetron sputtering. The stack is annealed in vacuum for $1 \mathrm{~h}$ at a temperature of $200^{\circ} \mathrm{C}$. Characterization of the material can be found in Ref. [6], where skyrmion diffusion is established in nominally identical samples. We obtain a typical hourglassshaped hysteresis loop, indicating the presence of a magnetic stripe or skyrmion phase [29]. A low effective anisotropy and saturation magnetization allow substantial changes in the domain alignment by small magnetic field changes.

By changing the applied magnetic out-of-plane (OOP) field, we can stabilize skyrmions and tune the skyrmion diameter, which we set to about $1.5 \mu \mathrm{m}$ by fixing the OOP field to $0.3 \mathrm{mT}$ throughout the whole experiment. The topologically nontrivial character of the skyrmions is checked in Ref. [6] by displacement via spin-orbit torques [16]. To image the diffusion dynamics, we use a magnetooptical Kerr effect (MOKE) microscope with a time resolution of $62.5 \mathrm{~ms}$. To achieve significant diffusion that is still detectable within the given time resolution, we set the sample temperature to $303 \mathrm{~K}$ using a Peltier element, leading to an increased diffusion coefficient compared with room temperature [6]. We use ImageJ's TrackMate plugin [30] to identify and track skyrmions during their diffusive motion (more details of the measurement setup and skyrmion tracking can be found in Appendix A1). The hereby-obtained skyrmion tracks are then used to investigate the time dependence of the mean-squared displacement that is fitted using a linear dependence to obtain the diffusion coefficients [6].

\section{RESULTS}

\section{A. Anisotropic skyrmion diffusion}

To go beyond the previous observation of isotropic diffusion [6], we need to break the in-plane symmetry. To this end, we probe the influence of a magnetic in-plane field along a selected direction on the diffusion properties. The magnetic in-plane field is applied additionally to the OOP field necessary to stabilize the skyrmion, leading effectively to a canted magnetic field. To visualize the effect, we assume the applied field direction as the $x$ axis and we now separately show in Fig. 1 the projected experimental mean-squared displacement (MSD),

$$
\left\langle x^{2}\right\rangle=\left\langle x(t)^{2}\right\rangle=2 D_{x} t
$$

parallel $\left(\left\langle x^{2}\right\rangle\right)$ and perpendicular $\left(\left\langle y^{2}\right\rangle\right)$ to the $x$-axis IP field as a function of time.

First, we observe that, at zero IP field, the MSDs parallel and perpendicular to the IP-field axis (solid lines) overlap within the error bars (standard error displayed by the shaded areas), as expected for the case of isotropic diffusion.

Next, we study the effect of the IP field systematically by measuring the diffusive motion for several IP-field values. We estimate the diffusion coefficient parallel $\left(D_{x}\right)$ and perpendicular $\left(D_{y}\right)$ to the IP-field axis, as shown in Fig. 2, by fitting the MSD displayed in Fig. 1 with a linear fit, according to Eq. (1).

The first striking observation is that, upon magnetic IP-field application, a significant decrease of the absolute

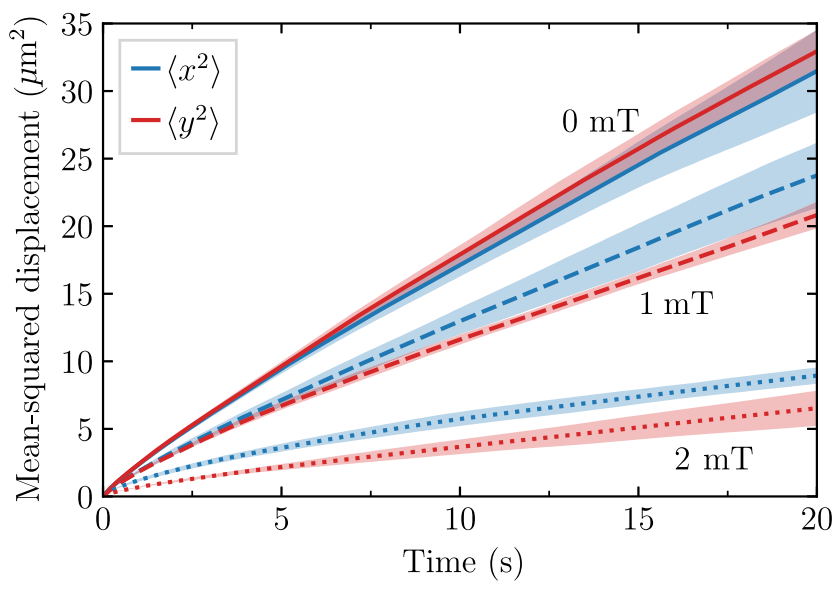

FIG. 1. Experimental mean-squared displacement under application of magnetic IP fields. $\left\langle x^{2}\right\rangle$ (blue) and $\left\langle y^{2}\right\rangle$ (red) as a function of time under application of a magnetic in-plane field of $0 \mathrm{mT}$ (solid line), $1 \mathrm{mT}$ (dashed line), and $2 \mathrm{mT}$ (dotted line). While the displacement without IP-field application shows isotropic diffusion within the error bars (standard error displayed by the shaded areas), application of a magnetic IP field leads to increasing anisotropic behavior. 
(a)

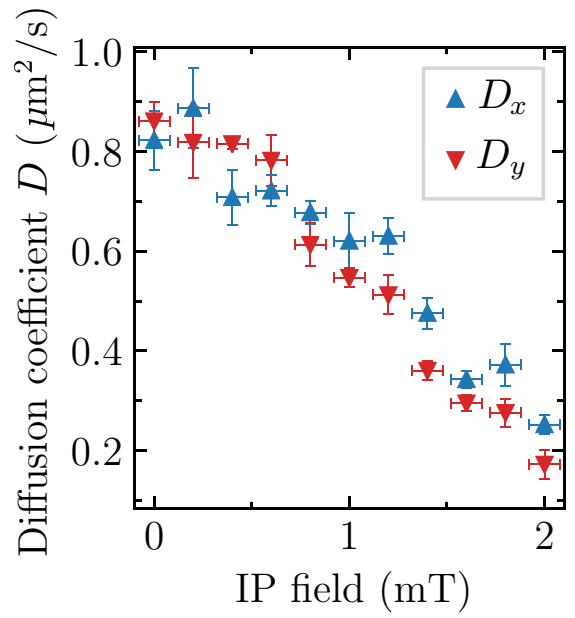

(b)

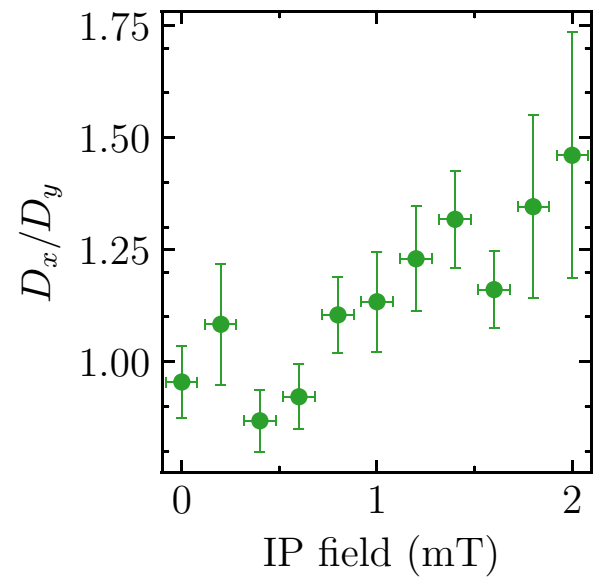

FIG. 2. Experimental diffusion coefficients under application of IP fields. (a) Diffusion coefficients $D_{x}$ and $D_{y}$ as a function of magnetic in-plane field. Application of an IP field leads to a decrease in diffusion coefficients, as well as (b) an increase of the ratio of the diffusion coefficients $D_{x} / D_{y}$ identifying anisotropic diffusion. value of the diffusion coefficient occurs [Fig. 2(a)]. Therefore, as a key result, we can tune the magnitude of the diffusion coefficient over a sizeable range dynamically, while previous handles to change the diffusion coefficient, such as temperature [6], are less practical as a fast and flexible control parameter.

As the second key result, we see very different diffusive behavior parallel and perpendicular to the IP-field axis [Fig. 2(a)]. The ratio of the diffusion coefficients, $D_{x} / D_{y}$, is shown in Fig. 2(b). Starting at a ratio of one, indicating isotropic diffusion at zero IP field, we find that, above a threshold field of about $0.8 \mathrm{mT}$, the ratio starts to increase up to a value of $1.46 \pm 0.27$ at the maximum IP field of $2 \mathrm{mT}$ used in this study. The threshold field value is given by the in-plane field, above which the anisotropy ratios of the diffusion coefficients are larger than one (outside the error bars). For even higher IP fields, the skyrmions in these samples are destabilized by the IP field and start to break up into stripes, so that we cannot investigate skyrmion diffusion in this regime (see Video S1 within the Supplemental Material [31]). We can exclude a change of the topological character of the skyrmions due to IP-field application, by checking their current-induced dynamics. We observe, also under the additional application of the magnetic IP fields, spin-orbit-torque-induced synchronous displacement of the skyrmions, indicating a topological charge $Q=1$ for the skyrmions, since $Q=0$ structures would either shrink or expand under current injection [32]. Additionally, a change in topology from $Q=1$ to $Q=0$ would lead to an expected drastic jump (increase) in the diffusion constant [33], instead of the continuous decrease observed in the experiment.

We therefore find that, by applying a magnetic in-plane field in a selected direction, we can generate anisotropic diffusion. This provides a means to steer thermal skyrmion motion. This is interesting, not just from a fundamental science perspective, but also for potential applications, where, in previous studies, the authors have patterned a skyrmion circuit geometrically to steer diffusive skyrmion motion $[22,24]$.

\section{B. Anisotropic skyrmion shape}

As the next step, we need to understand the origin of the observed anisotropic diffusion dynamics. Fundamentally, there are different possible origins, as the in-plane field changes, for example, the skyrmion shape, the domain wall delineating the skyrmion, and the average in-plane magnetization in the sample. In the field of soft matter, it is known that particles with an anisotropic shape, such as elongation, show anisotropic diffusion with different diffusion coefficients along the long and short axes [27,28]. Drawing on this experience, we study the shape of the skyrmion and determine the skyrmion diameter size parallel and perpendicular to the IP-field axis. We fit each tracked skyrmion in each frame by a two-dimensional (2D) Gaussian function:

$f(x, y)=A \exp \left\{-\left[\left(\frac{\left(x-x_{0}\right)^{2}}{2 \sigma_{x}^{2}}\right)+\left(\frac{\left(y-y_{0}\right)^{2}}{2 \sigma_{y}^{2}}\right)\right]\right\}$,

with the size $s_{X}=2 \sqrt{2 \ln (2)} \sigma_{x}$ parallel and $s_{Y}=$ $2 \sqrt{2 \ln (2)} \sigma_{y}$ perpendicular to the IP-field axis given by the FWHM, as indicated in Fig. 3(a) by a red ellipse. We note that, due to diffusive motion, as well as the influence of pinning sites, the skyrmion shape might be distorted in single frames, while the small error bars presented in Fig. 3(b) are based on the statistics from averaging over all skyrmions and frames. Averaging over all frames leads to the field dependence of the skyrmion size shown in Fig. 3(b). While the size perpendicular to the field direction only slightly decreases with increasing IP field, one can observe a sizable increase of the skyrmion size above a field of about $1 \mathrm{mT}$, as shown in Fig. 3(b). This, in turn, leads to an increase of the size ratio above a threshold IP field of about $1 \mathrm{mT}$, as shown in Fig. 3(c). We note that 
(a)

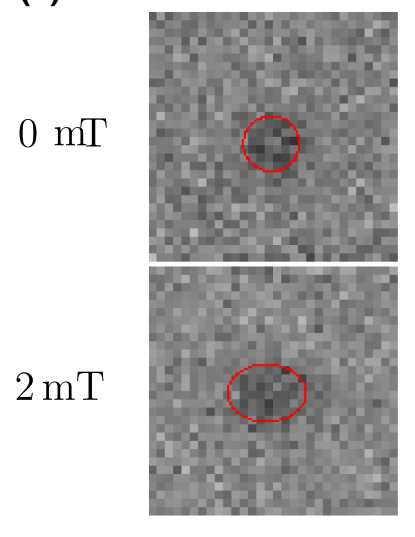

(b)

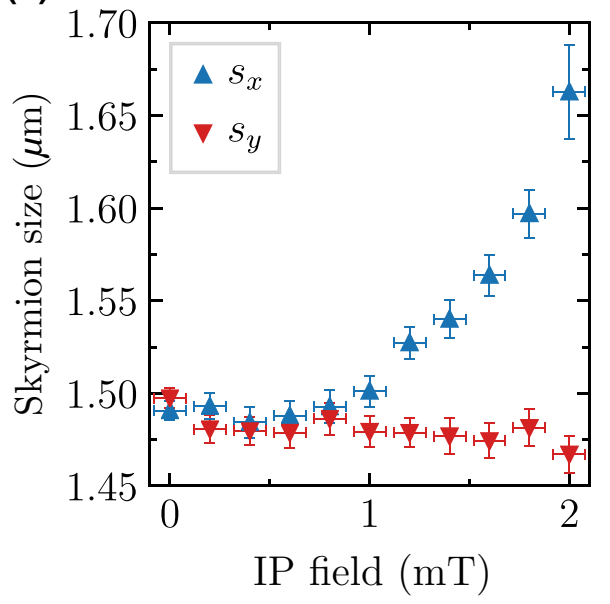

(c)

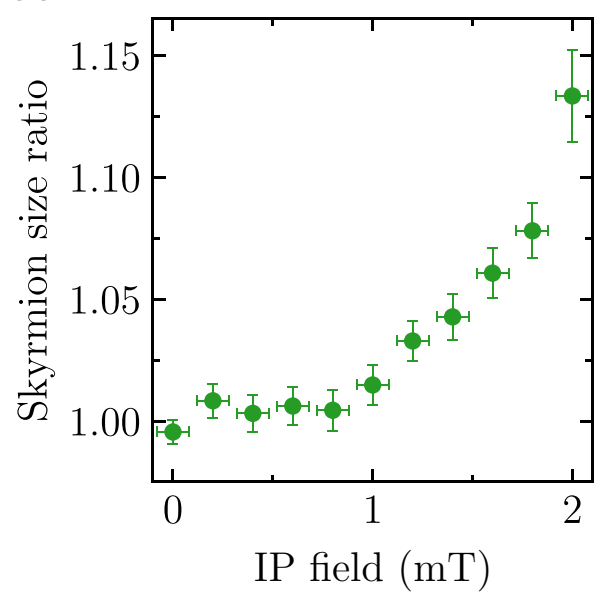

FIG. 3. Experimentally determined skyrmion size under application of IP fields. (a) Single frames of the experimental skyrmion profile at 0 and $2 \mathrm{mT}$ in-plane field. Red ellipse indicates the FWHM of the fitted 2D Gaussian function. (b) Skyrmion size, $s_{x}$ and $s_{y}$, as a function of magnetic in-plane field. Application of an IP field leads to elongation of the skyrmion parallel to the field axis. (c) This subsequently leads to an increase of the size ratio $s_{x} / s_{y}$ upon IP-field application.

the threshold field for the skyrmion size is in line with the observed threshold field for diffusion coefficients.

As a next step, we have to thus check theoretically if our results for anisotropic shape are expected to lead to anisotropic diffusion.

\section{DISCUSSION}

\section{A. Analytical derivation of the diffusion tensor}

To investigate theoretically the influence of the skyrmion shape on the diffusive dynamics of skyrmions, we start with an analytical model of skyrmion diffusion. In a theoretical study [20] of thermal skyrmion diffusion, the following expression for the diffusion coefficient of a rotationally symmetric skyrmion in a rotationally and translationally invariant two-dimensional film was derived from Thiele's equation [34]:

$$
D^{\text {iso }}=k_{B} T \frac{\alpha D_{T}}{\left(\alpha D_{T}\right)^{2}+G^{2}}
$$

with a gyrocoupling of $G=M_{2 d} / \gamma \int \mathbf{S} \cdot\left(\partial_{x} \mathbf{S} \times \partial_{y} \mathbf{S}\right)$ $d^{2} r=4 Q M_{2 d} / \gamma$, and $2 D_{T}$ being the trace over the dissipative tensor $D_{i j}=M_{2 d} / \gamma \int \partial_{i} \mathbf{S} \partial_{j} \mathbf{S} d^{2} r$ and $M_{2 d}=M_{s} / d$ being the saturation magnetization divided by the thickness of the magnetic layer. Notably, only the dissipative tensor depends on the skyrmion profile, whereas gyrocoupling is a topological invariant. Equation (3) describes isotropic diffusive motion with $\left\langle x^{2}\right\rangle=\left\langle y^{2}\right\rangle=2 D^{\text {iso }} t$ and its validity is demonstrated via atomistic spin simulations $[6,33,35]$.

However, if the rotational symmetry of the magnetic texture is broken, the dissipation tensor becomes anisotropic, which consequently leads to different dynamics along different axes of the skyrmion. For current-driven motion of spin structures with broken rotational symmetry, the anisotropy of the skyrmion dynamics was recently predicted [36,37].

As anisotropic diffusion dynamics is not yet treated in the literature, we derive the following generalization of Eq. (3) for a general dissipative tensor starting from Thiele's equation (shown in Appendix B), yielding the diffusion tensor:

$$
\begin{aligned}
D= & {\left[\begin{array}{cc}
\left\langle x^{2}(t)\right\rangle & \langle x y(t)\rangle \\
\langle x y(t)\rangle & \left\langle y^{2}(t)\right\rangle
\end{array}\right] / 2 t=\frac{k_{B} T}{\alpha^{2}\left(D_{x x} D_{y y}-D_{x y}^{2}\right)+G^{2}} } \\
& \times\left(\begin{array}{cc}
\alpha D_{y y} & -\alpha D_{x y} \\
-\alpha D_{x y} & \alpha D_{x x}
\end{array}\right) .
\end{aligned}
$$

As the diffusion tensor and the dissipation tensor are both diagonalizable and connected via Eq. (4), we choose, for the following discussion, a coordinate system for which the off-diagonal terms in both tensors vanish, i.e., $\langle x y(t)\rangle=0$. For simplicity, the diagonal elements of the diffusion tensor are referred to as $D_{x}$ and $D_{y}$ in what follows, with $\left\langle x^{2}(t)\right\rangle=$ $2 D_{x} t$ and $\left\langle y^{2}(t)\right\rangle=2 D_{y} t$.

The anisotropy of diffusive motion can most readily be characterized by the ratio $D_{x} / D_{y}$.

Equation (4) predicts that this ratio can be expressed as $D_{y y} / D_{x x}$, which implies anisotropic diffusion for spin textures with $D_{y y} \neq D_{x x}$. In most systems, skyrmions are found to be of circular shape, resulting in isotropic behavior with $D_{x x}=D_{y y}$. However, it has been recently demonstrated $[36,38]$ that an applied in-plane field can lead to an anisotropic skyrmion profile. When we now feed this into 
our generalized calculation, this consequently leads to different dissipation tensor elements for the directions along the in-plane field and orthogonal to it.

\section{B. Micromagnetic simulations}

As the deformation can be continuously tuned by varying the strength of the applied in-plane field, this should, according to the theoretical predictions derived above, allow for a transition from isotropic to anisotropic diffusive motion. While we can thus qualitatively understand the experimental results, we next test the analytically derived dependence of skyrmion diffusion on the skyrmion profile by micromagnetic simulations using MUMAX [39]. First, the skyrmion profile is equilibrated under the application of different IP fields (more details about the micromagnetic simulations can be found in Appendix A2). The size parallel ( $x$ axis) and perpendicular ( $y$ axis) to the IP-field axis is shown in Fig. 4(b). The skyrmion elongates strongly parallel and shortens slightly perpendicular to the IP field above a certain threshold field of about $0.75 \mathrm{mT}$. We here find not only qualitative agreement, but also quantitative agreement with the experimentally observed behavior, when the parameters used are also comparable to those obtained experimentally. The size ratio $s_{x} / s_{y}$ shown in Fig. 4(c) reproduces the experimental results, with flat behavior up to a threshold of about $0.75 \mathrm{mT}$ followed by an increase of this ratio.

Next, the simulated skyrmion profile is used to numerically calculate the dissipation tensor elements $D_{x x}$ and $D_{y y}$ to obtain quantitative insights into the expected anisotropy of diffusion. Since the dissipation tensor elements are directly connected to the diffusion coefficients $D_{x}$ and $D_{y}$, as derived above, we directly calculate these as presented in Fig. 5(a), where we assume that $\alpha=0.01$. Based on the obtained size-field dependence, we obtain, above the threshold field of $0.75 \mathrm{mT}$, a slightly increased diffusion parallel to the IP-field axis, while the diffusion coefficients decrease strongly perpendicular to the IP field. We find that the numerically obtained ratio, $D_{x} / D_{y}$, of the diffusion coefficients shown in Fig. 5(b) agrees with the experimental results.

Thus, by comparing theory and experiment, we identify that the origin of the observed anisotropic diffusion induced by an IP field is the deformation of the skyrmion shape due to this field. Analytical calculations reproduce the trend that nonspherical skyrmions lead to anisotropic diffusion due to nonsymmetric dissipation tensor elements, and numerical calculations allow us to quantitatively reproduce the experimentally found skyrmion shape deformation, which, when entered into the analytical calculation, reproduces the anisotropy of diffusion quantitatively with good fidelity.

We want to point out that we do not exclude possible additional contributions by in-plane field-induced changes of the domain wall (width) or the average in-plane magnetization in the sample. By calculating numerically the dissipation tensor elements for the numerically simulated skyrmions, the combined influence of all factors are entered automatically into the presented numerical diffusion coefficients. While slight changes in the domain-wall width upon IP-field application can be observed in the micromagnetic simulations (roughly $2.5 \%$ at $2 \mathrm{mT}$ ), their influence on the dissipation tensor elements (and thus, the diffusion coefficients) is small compared with the influence due to the change of the skyrmion's size (about $10 \%$ at (a)

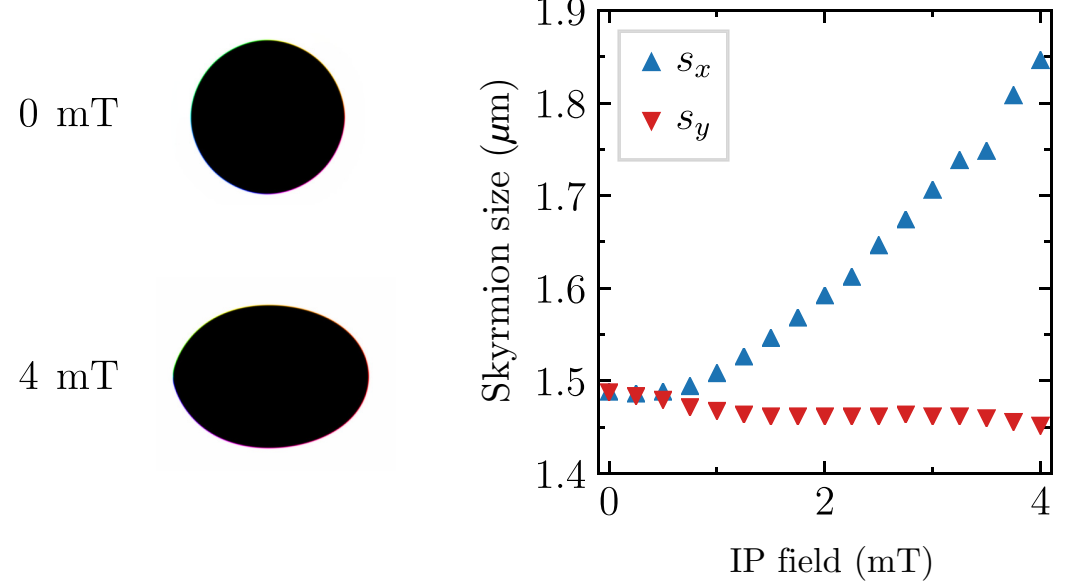

(c)

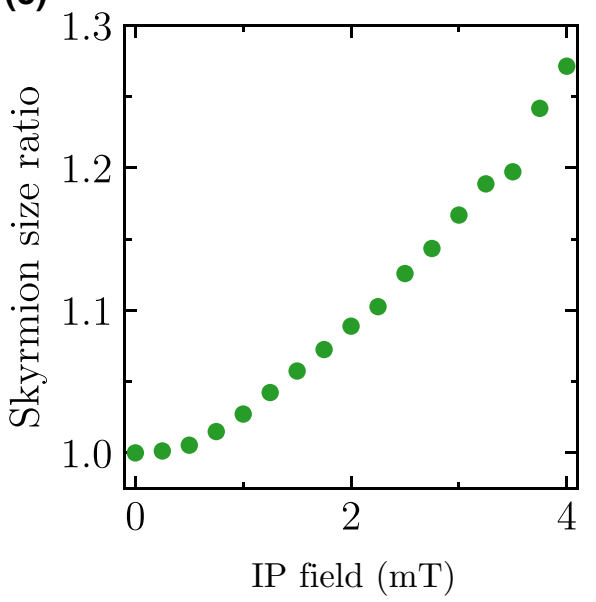

FIG. 4. Numerically simulated skyrmion size under application of IP fields. (a) Numerically simulated skyrmion profile at 0 and $4 \mathrm{mT}$ in-plane field. (b) Numerical skyrmion size, $s_{x}$ and $s_{y}$, as a function of magnetic in-plane field. Application of an IP field leads to elongation of the skyrmion parallel to the field axis and a decrease perpendicular to it. (c) This, in turn, increases the size ratio $s_{x} / s_{y}$ upon IP-field application. 


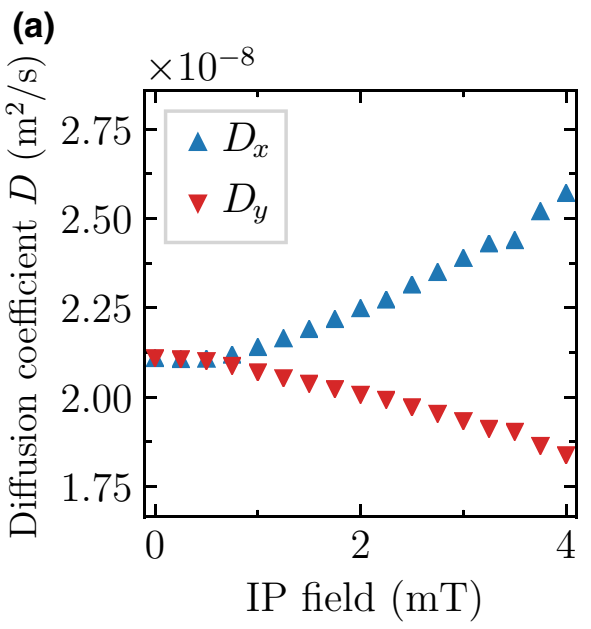

(b)

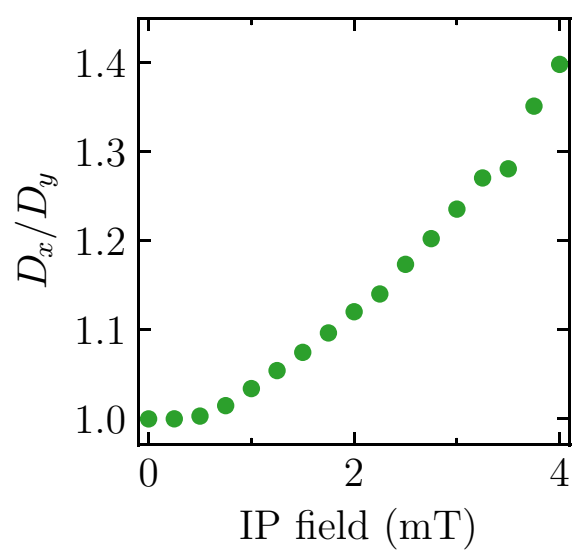

FIG. 5. Analytically calculated diffusion coefficients under application of IP fields. (a) Calculated diffusion coefficients $D_{x}$ and $D_{y}$, following Eq. (4), using numerically determined dissipative tensor elements as a function of magnetic in-plane field. Application of an IP field leads to an increase of the diffusion coefficient parallel to the IPfield axis and a decrease perpendicular to it. (b) Increase of the ratio of diffusion coefficients $D_{x} / D_{y}$, and therefore, anisotropic diffusion upon IP-field application follows.
$2 \mathrm{mT}$ ). This is also reflected in the fact that the numerical skyrmion size ratio and the calculated diffusion coefficient ratio are strongly correlated.

Finally, we note that our theory does not reproduce all details of the experimental results. In particular, we are not able to reproduce the observed strong decreases of both diffusion coefficients upon IP-field application. This means that there needs to be an additional mechanism, which scales both diffusion coefficients in a similar fashion, leading to a strong decrease of both coefficients upon IP-field application, while preserving the anisotropic diffusion ratio. A possible source of this discrepancy is the fact that, theoretically, we consider free skyrmion diffusion in a flat energy-potential landscape. In a nonflat energy-potential landscape, it is shown that the influence of pinning sites leads to a clear exponential dependence of the diffusion coefficient on temperature, and thus, to a deviation from the pinning-free case [6]. As pinning of the skyrmions can be affected by changes to the shape and size, this can then affect the absolute values of the diffusion coefficients. Details of this are, however, beyond the scope of the current investigation and could be studied in future work.

\section{CONCLUSION}

Using time-resolved MOKE microscopy, we observe skyrmion diffusion, the magnitude and anisotropy of which we can control by the strength and direction of an applied IP-field. We identify a dependence of the anisotropy of the skyrmion shape on the IP field that is similar to the field dependence of diffusion anisotropy. To understand this, we perform analytical calculations, from which we obtain a connection between the diffusion tensor and the dissipation tensor, which depends on the skyrmion shape. Carrying out additional micromagnetic simulations and numerical calculations, we obtain qualitative and quantitative agreement with the experimentally observed behavior. Thus, our findings reveal how, by tuning the shape of the skyrmion with a magnetic field, we are able to control the diffusive dynamics. Given the fact that the skyrmion shape can be controlled by a variety of additional means, including dynamic strain, and strongly elliptical skyrmions are observed [40], our findings open up a range of possible handles to control diffusive dynamics. It is exactly this type of control that will enable the implementation of skyrmion-diffusionbased applications, such as the proposed nonconventional logic [21].

\section{ACKNOWLEDGMENTS}

N.K., K.L., and M.K. gratefully acknowledge financial support by the Graduate School of Excellence Materials Science in Mainz (MAINZ, Grant No. GSC266). The Deutsche Forschungsgemeinschaft (DFG, German Research Foundation) funds this project in Mainz and Konstanz through the priority program SPP 2137 Skyrmionics (No. 403502522), the group in Konstanz via SFB1432 and M.K. and the group in Mainz through Projects No. 268565370/TRR173 and support from the Horizon 2020 Framework Programme of the European Commision is acknowledged (Grant No. 856538 3D MAGiC). J.Z. acknowledges support from Charles University (Grant No. PRIMUS/20/SCI/018).

\section{APPENDIX A: METHODS}

\section{Measurement setup and skyrmion tracking}

The measurement setup consists of a commercial Evico $\mathrm{GmbH}$ MOKE full-field microscope that is used to image skyrmion diffusion with a camera recording 16 frames per second, and therefore, a time resolution of $62.5 \mathrm{~ms}$. The sample temperature is set to $303 \mathrm{~K}$ using a Peltier QC-32-0.6-1.2 element and controlled by a resistivity measurement on a Pt100 resistor. The in-plane field coil from Evico $\mathrm{GmbH}$ is aligned to generate a field perpendicular to a custom-made out-of-plane field coil. The precise perpendicular alignment is achieved by taking out-of-plane 
hysteresis loops with and without an applied magnetic in-plane field to ensure that the applied in-plane field contributes no out-of-plane field component that will lead to a horizontal shift of the out-of-plane hysteresis loop.

To identify and track the skyrmion motion, ImageJ's TrackMate plugin [30] is used. Videos S2 and S3 within the Supplemental Material visualize the skyrmion trajectories of a measurement at an in-plane field of 0 and $2 \mathrm{mT}$ [41]. At each in-plane field, several measurements containing several skyrmions are performed to calculate the averaged MSD. Furthermore, to obtain better statistics, the time-averaged MSD is determined, averaging the MSDs of every starting point over the whole video, leading effectively to improved statistics, in particular, for short timescales. The statistical error of the MSD therefore increases with time. The error bars of the averaged MSD at a given in-plane field, shown in Fig. 1 by the shaded area, are given by the standard error of the averaged MSD. The diffusion coefficients in Fig. 2 are obtained by a linear fit. The error of the diffusion coefficients is obtained from the standard error of the MSDs.

\section{Micromagnetic simulation}

Micromagnetic simulations are performed using the simulation program MUMAX [39]. The following magnetic material parameters are used in the simulations: Dzyaloshinskii-Moriya interaction $\left(D=0.0985 \mathrm{~mJ} / \mathrm{m}^{2}\right)$, exchange stiffness constant $\left(A=2.4610^{-12} \mathrm{~J} / \mathrm{m}\right)$, saturation magnetization $\left(M_{S}=9.810^{5} \mathrm{~A} / \mathrm{m}\right)$, and perpendicular magnetic anisotropy $\left(K_{\text {eff }}=6.4 \times 10^{4} \mathrm{~J} / \mathrm{m}^{3}\right)$. The skyrmion profile is equilibrated under the application of different magnetic IP fields at a fixed magnetic OOP field of $0.2 \mathrm{mT}$. The size parallel ( $x$ axis) and perpendicular ( $y$ axis) to the IP-field axis is determined and is displayed in Fig. 4. The simulated skyrmion profile is used to numerically calculate the dissipation tensor elements $D_{x x}$ and $D_{y y}$ that are used to calculate the diffusion tensor elements, as described in Eq. (B3).

\section{APPENDIX B: ANALYTICAL DERIVATION OF THE DIFFUSION TENSOR}

Thiele's equation describes the movement of a rigid magnetic texture and reads as $G v+\alpha D v=F$, where $G v$ is the gyrotropic force, $\alpha D v$ is the dissipative force, and $F$ is an applied force. In the case of thermal diffusion, the applied force is a fluctuating stochastic force, leading to a Brownian-type motion of the skyrmion.

In the so-called "low-noise" limit, its autocorrelation is given via [42]

$$
\left\langle F_{i}(t) F_{j}\left(t^{\prime}\right)\right\rangle=2 \alpha k_{B} T D_{i j} \delta\left(t-t^{\prime}\right) .
$$

By expressing the cross product in the gyrotropic force via a skew-symmetric matrix skew $[G]$, Thiele's equation can be rewritten as

$$
v=(\operatorname{skew}[G]+\alpha D)^{-1} F,
$$

which, together with Eq. (B1), allows for the calculation of the velocity autocorrelation function $\left\langle v_{i}(t) v_{j}\left(t^{\prime}\right)\right\rangle$. Finally, time integration, $r(t)=\int_{0}^{t} v(\tau) d \tau$, for the diffusion tensor yields

$$
\begin{aligned}
D= & {\left[\begin{array}{cc}
\left\langle x^{2}(t)\right\rangle & \langle x y(t)\rangle \\
\langle x y(t)\rangle & \left\langle y^{2}(t)\right\rangle
\end{array}\right] / 2 t=\frac{k_{\mathrm{B}} T}{\alpha^{2}\left(D_{x x} D_{y y}-D_{x y}^{2}\right)+G^{2}} } \\
& \times\left(\begin{array}{cc}
\alpha D_{y y} & -\alpha D_{x y} \\
-\alpha D_{x y} & \alpha D_{x x}
\end{array}\right) .
\end{aligned}
$$

[1] A. Fert, V. Cros, and J. Sampaio, Skyrmions on the track, Nat. Nanotechnol. 8, 152 (2013).

[2] X. Zhang, M. Ezawa, and Y. Zhou, Magnetic skyrmion logic gates: Conversion, duplication and merging of skyrmions, Sci. Rep. 5, 9400 (2015).

[3] Y. Huang, W. Kang, X. Zhang, Y. Zhou, and Weisheng Zhao, Magnetic skyrmion-based synaptic devices, Nanotechnology 28, 08LT02 (2017).

[4] D. Pinna, F. Abreu Araujo, J.-V. Kim, V. Cros, D. Querlioz, P. Bessiere, J. Droulez, and J. Grollier, Skyrmion Gas Manipulation for Probabilistic Computing, Phys. Rev. Appl. 9, 064018 (2018).

[5] A. Fert, N. Reyren, and V. Cros, Magnetic skyrmions: Advances in physics and potential applications, Nat. Rev. Mater. 2, 17031 (2017).

[6] J. Zázvorka, F. Jakobs, D. Heinze, N. Keil, S. Kromin, S. Jaiswal, K. Litzius, G. Jakob, P. Virnau, D. Pinna, K. Everschor-Sitte, L. Rózsa, A. Donges, U. Nowak, and M. Kläui, Thermal skyrmion diffusion used in a reshuffler device, Nat. Nanotechnol. 14, 658 (2019).

[7] I. Dzyaloshinsky, A thermodynamic theory of "weak" ferromagnetism of antiferromagnetics, J. Phys. Chem. Solids 4, 241 (1958).

[8] T. T. Moriya, Anisotropic superexchange interaction and weak ferromagnetism, Phys. Rev. 120, 91 (1960).

[9] U. K. Rößler, A. N. Bogdanov, and C. Pfleiderer, Spontaneous skyrmion ground states in magnetic metals, Nature 442, 797 (2006).

[10] S. Mühlbauer, B. Binz, F. Jonietz, C. Pfleiderer, A. Rosch, A. Neubauer, R. Georgii, and P. Böni, Skyrmion lattice in a chiral magnet, Science 323, 915 (2009).

[11] X. Z. Yu, N. Kanazawa, Y. Onose, K. Kimoto, W. Z. Zhang, S. Ishiwata, Y. Matsui, and Y. Tokura, Extended skyrmion phase in epitaxial FeGe(111) thin films, Nat. Mater. 10, 106 (2011).

[12] K. Everschor-Sitte, J. Masell, R. M. Reeve, and M. Kläui, Overview of recent progress in an active research field, J. Appl. Phys. 124, 240901 (2018).

[13] W. Jiang, G. Chen, K. Liu, J. Zang, S. G. E. te Velthuis, and A. Hoffmann, Skyrmions in magnetic multilayers, Phys. Rep. 704, 1 (2017). 
[14] R. Wiesendanger, Nanoscale magnetic skyrmions in metallic films and multilayers: A new twist for spintronics, Nat. Rev. Mater. 1, 16044 (2016).

[15] S. Finizio, K. Zeissler, S. Wintz, S. Mayr, T. Weßels, A. J. Huxtable, G. Burnell, C. H. Marrows, and J. Raabe, Deterministic field-free skyrmion nucleation at a nanoengineered injector device, Nano Lett. 19, 7246 (2019).

[16] W. Jiang, Z. Xichao, G. Yu, W. Zhang, X. Wang, M. B. Jungfleisch, J. E. Pearson, X. Cheng, O. Heinonen, K. L. Wang, Y. Zhou, A. Hoffmann, and S. G. E. te Velthuis, Direct observation of the skyrmion Hall effect, Nat. Phys. 13, 162 (2017).

[17] S. Woo, K. Litzius, B. Krüger, M.-Y. Im, L. Caretta, K. Richter, M. Mann, A. Krone, R. M. Reeve, M. Weigand, P. Agrawal, I. Lemesh, M.-A. Mawass, P. Fischer, M. Kläui, and G. S. D. Beach, Observation of room-temperature magnetic skyrmions and their current-driven dynamics in ultrathin metallic ferromagnets, Nat. Mater. 15, 501 (2016).

[18] K. Litzius, I. Lemesh, B. Krüger, P. Bassirian, L. Caretta, K. Richter, F. Büttner, K. Sato, O. A. Tretiakov, J. Förster, R. M. Reeve, M. Weigand, I. Bykova, H. Stoll, G. Schütz, G. S. D. Beach, and M. Kläui, Skyrmion hall effect revealed by direct time-resolved X-ray microscopy, Nat. Phys. 13, 170 (2017).

[19] K. Litzius, J. Leliaert, P. Bassirian, D. Rodrigues, S. Kromin, I. Lemesh, J. Zazvorka, K.-J. Lee, J. Mulkers, N. Kerber, D. Heinze, N. Keil, R. M. Reeve, M. Weigand, B. V. Waeyenberge, G. Schütz, K. Everschor-Sitte, G. S. D. Beach, and M. Kläui, The role of temperature and drive current in skyrmion dynamics - A route towards universality in skyrmion Hall angle and mobility, Nat. Electronics 3, 30 (2020).

[20] C. Schütte, J. Iwasaki, A. Rosch, and N. Nagaosa, Inertia, diffusion, and dynamics of a driven skyrmion, Phys. Rev. B 90, 174434 (2014).

[21] T. Nozaki, Y. Jibiki, M. Goto, E. Tamura, T. Nozaki, H. Kubota, A. Fukushima, S. Yuasa, and Y. Suzuki, Brownian motion of skyrmion bubbles and its control by voltage applications, Appl. Phys. Lett. 114, 012402 (2019).

[22] Y. Jibiki, M. Goto, E. Tamura, J. Cho, H. Nomura, T. Srivastava, W. Lim, S. Auffret, C. Baraduc, H. Bea, and Y. Suzuki, Skyrmion Brownian circuit implemented in a continuous ferromagnetic thin film, Appl. Phys. Lett. 117, 082402 (2020).

[23] L. Zhao, Z. Wang, X. Zhang, J. Xia, K. Wu, H.-A. Zhou, Y. Dong, G. Yu, K. L. Wang, X. Liu, Y. Zhou, and W. Jiang, Topology-Dependent Brownian Gyromotion of a Single Skyrmion, Phys. Rev. Lett. 125, 027206 (2020).

[24] F. Peper, J. Lee, J. Carmona, J. Cortadella, and K. Morita, Brownian circuits: Fundamentals, ACM J. Emerg. Technol. Comput. Syst. 9, 3 (2013).

[25] E. Nelson, Dynamical Theories of Brownian Motion (Princeton Univ. Press, Princeton, NJ, 1972).

[26] A. Einstein, Über die von der molekularkinetischen Theorie der Wärme geforderte Bewegung von in ruhenden Flüssigkeiten suspendierten Teilchen, Ann. Phys. 17, 549 (1905).
[27] J. Happel and H. Brenner, Low Reynolds Number Hydrodynamics (Kluwer, Dordrecht, Netherlands, 1991).

[28] Y. Han, A. M. Alsayed, M. Nobili, J. Zhang, T. C. Lubensky, and A. G. Yodh, Brownian motion of an ellipsoid, Science 314, 626 (2006).

[29] I. Lemesh, K. Litzius, M. Böttcher, P. Bassirian, N. Kerber, D. Heinze, J. Zázvorka, F. Büttner, L. Caretta, M. Mann, M. Weigand, S. Finizio, J. Raabe, M-Y Im, Hermann Stoll, G. Schütz, B. Dupé, M. Kläui, and G. S. D. Beach, Current-Induced skyrmion generation through morphological thermal transitions in chiral ferromagnetic heterostructures, Adv. Mater. 30, 1805461 (2018).

[30] J.-Y. Tinevez, N. Perry, J. Schindelin, G. M. Hoopes, G. D. Reynolds, E. Laplantine, S. Y. Bednarek, S. L. Shorte, and K. W. Eliceiri, Trackmate: An open and extensible platform for single-particle tracking, Methods 115, 80 (2017).

[31] See the Supplemental Material at http://link.aps.org/supple mental/10.1103/PhysRevApplied.15.044029 for the creation of aligned magnetic stripe domains in a $4 \mathrm{mT}$ magnetic in-plane field.

[32] W. Jiang, P. Upadhyaya, W. Zhang, G. Yu, M. B. Jungfleisch, F. Y. Fradin, J. E. Pearson, Y. Tserkovnyak, K. L. Wang, O. Heinonen, S. G. E. te Velthuis, and Axel Hoffmann, Blowing magnetic skyrmion bubbles, Science 349, 283 (2015).

[33] M. Weißenhofer and U. Nowak, Diffusion of skyrmions: The role of topology and anisotropy, New J. Phys. 22, 103059 (2020).

[34] A. A. Thiele, Steady-State Motion of Magnetic Domains, Phys. Rev. Lett. 30, 230 (1973).

[35] J. Miltat, S. Rohart, and A. Thiaville, Brownian motion of magnetic domain walls and skyrmions, and their diffusion constants, Phys. Rev. B 97, 214426 (2018).

[36] M. Weißenhofer and U. Nowak, Orientation-dependent current-induced motion of skyrmions with various topologies, Phys. Rev. B 99, 224430 (2019).

[37] J. Xia, X. Zhang, M. Ezawa, Q. Shao, X. Liu, and Y. Zhou, Dynamics of an elliptical ferromagnetic skyrmion driven by the spin-orbit torque, Appl. Phys. Lett. 116, 022407 (2020).

[38] S.-Z. Lin and A. Saxena, Noncircular skyrmion and its anisotropic response in thin films of chiral magnets under a tilted magnetic field, Phys. Rev. B 92, 180401(R) (2015).

[39] A. Vansteenkiste, J. Leliaert, M. Dvornik, M. Helsen, F. Garcia-Sanchez, and B. V. Waeyenberge, The design and verification of MuMax3, AIP Adv 4, 107133 (2014).

[40] J. Jena, B. Göbel, T. Ma, V. Kumar, R. Saha, I. Mertig, C. Felser, and S. S. P. Parkin, Elliptical Bloch skyrmion chiral twins in an antiskyrmion system, Nat. Commun. 11, 1115 (2020).

[41] See the Supplemental Material at http://link.aps.org/supple mental/10.1103/PhysRevApplied.15.044029 for skyrmion diffusion in 0 and $2 \mathrm{mT}$ magnetic in-plane fields.

[42] T. Kamppeter, F. G. Mertens, E. Moro, A. Sánchez, and A. R. Bishop, Stochastic vortex dynamics in two-dimensional easy-plane ferromagnets: Multiplicative versus additive noise, Phys. Rev. B 59, 11349 (1999). 